\title{
Editorial
}

\section{Internet de las cosas para el cuidado de la salud}

\section{Internet of things for health care}

\author{
Line Yasmin Becerra Sánchez, PhD.
}

La gran variedad de sensores y cosas inteligentes conectadas en los últimos años, hacen que Internet de las cosas (Internet of Things, IoT) sea un paradigma esencial en todos los campos. IoT es un concepto que refleja un conjunto conectado de cualquier persona, máquina, servicio o cosa, en cualquier momento, lugar y en cualquier red. IoT es una mega-tendencia en las tecnologías de próxima generación, proporcionando soluciones pertinentes para una amplia gama de aplicaciones como: ciudades inteligentes, congestión de tráfico, gestión de residuos, seguridad, servicios de emergencias, logística, control industrial y cuidado de la salud. Siendo esta última una de las principales áreas actuales que están siendo foco de investigación [1].

El paradigma principal de IoT, ha permitido varias derivaciones en el campo de la salud [2]. El internet portátil de las cosas (Wearable Internet of things, WIoT) [3], tiene la intención de implementar tele salud con el fin de formar un ecosistema de intervenciones médicas automatizadas, valiéndose del uso de sensores en el cuerpo lo que permite monitorear datos útiles para mejorar calidad de vida de las personas y conectar a los pacientes a infraestructuras médicas. La Internet de las cosas sanas (Internet of Health Things, IoHT) es otra derivación que se basa en la combinación de aplicaciones móviles, portátiles y otros dispositivos conectados aprovechando su comportamiento de estar siempre conectados (always on) [4]. Internet de las cosas médicas (Internet of Medical Things, IoMT) [5], se refiere a aplicaciones que consisten en dispositivos implantables y portátiles conectados a un teléfono o un reloj inteligentes que esten conectados a Internet y actuando como una central de actividad personal. El Internet de las nano cosas (Internet of Nano Things, IoNT) [6] se refiere a la aplicación de IoT en nanomedicina, para implementar monitoreo, diagnóstico y tratamientos más personalizados para implementar monitoreo proactivo, salud preventiva, atención al manejo de enfermedades crónicas de seguimiento. La Internet de cosas sanas móviles (Internet of mobile-health Things, $m$-IoT) [7] prevé un modelo de conectividad entre redes de área personal de baja potencia y evolución redes 4G, resaltando las características específicas existentes intrínsecas a la movilidad global de las entidades participantes [2].

Sin embargo, con el conocimiento de los avances mencionados, se puede deducir que IoT está apenas dando los primeros pasos en el campo de la atención médica [8]. El concepto de IoT consiste en la interconexión de objetos inteligentes, por tanto, este paradigma se enfrenta a grandes desafíos. Uno de los desafíos principales es la energía, este es un gran desafío técnico. De hecho, se necesita investigación sobre cosecha de energía, conservación de energía, energía y uso, para diseñar y desarrollar sistemas de cosecha de energía del ambiente y no desperdiciar nada en operación [2,9]. Otro desafío importante se refiere a la escalabilidad, teniendo en cuenta que los objetos interconectados superarán a los conectados en la Internet convencional (se espera que IoT este 
compuesto por trillones de dispositivos), y por otro lado, los servicios para el cuidado del salud se caracterizan por tener una alta demanda, es por esto que la escalabilidad es un asunto de preocupación actualmente [10, 11]. Finalmente, otro desafío que requiere mucha atención es lo correspondiente a seguridad y privacidad, se necesita más investigación para desarrollar y diseñar soluciones apropiadas de seguridad para IoT.

Se puede notar que la evolución de las tecnologías de información y las comunicaciones están transformando todas las actividades humanas, impactando fuertemente al sector de la salud. Frente a este panorama, la visión de futuro con respecto a las tecnologías de tendencia, enmarcadas en la industria 4.0, juegan un papel importante en los avances en el sector salud, es por esto que se habla de tecnologías 4.0 para cuidado de la salud (Health Care 4.0 o HC4.0). Este enfoque traerá muchos beneficios para los seres humanos. El impacto en el bienestar y en la calidad de vida en general, tanto para las personas sanas como para los pacientes enfermos, es más fácil de imaginar, ya que se podrán promover los buenos hábitos y los tratamientos oportunos mientras se reducen los costos de hospitalización y atención médica.

\section{REFERENCIAS}

[1] Riazul Islam, S. M., Daehan Kwak, Humaun Kabir, M.D., Mahmud Hossain, And Kyung-Sup Kwak, The Internet of Things for Health Care: A Comprehensive Survey. IEEE Access, vol. 3, 2015. DOI:10.1109/ACCESS.2015.2437951.

[2] Aceto, G., Persico, V. and Pescape, A. Industry 4.0 and Health: Internet of Things, Big Data, and Cloud Computing for Healthcare 4.0, Journal of Industrial Information Integration Volu K. me 18, June 2020. DOI: https://doi.org/10.1016/j.jii.2020.100129.

[3] Hiremath, S., Yang, G. and Mankodiya, K. Wearable internet of things: Concept, architectural components and promises for person-centered healthcare. In Wireless Mobile Communication and Healthcare (Mobihealth), 2014 EAI 4th International Conference on. IEEE, 2014.

[4] Terry, N. Will the internet of things disrupt healthcare? 2016.

[5] Jha, N. K. Internet-of-medical-things. In Proceedings of the on Great Lakes Symposium on VLSI 2017, GLSVLSI '17, New York, NY, USA, 2017. ACM.

[6] Omanovi'c-Mikli canin, E., Maksimovi'c, M. and Vujovi'c, V. The future of healthcare: Nanomedicine and internet of nano things. Folia Medica Facultatis Medicinae Universitatis Saraeviensis, 50(1), 2015.

[7] Istepanian, R. S. H., Hu, S., Philip, N. Y. and Sungoor, A. The potential of internet of m-health things "m-iot" for non-invasive glucose level sensing. In 2011 Annual International Conference of the IEEE Engineering in Medicine and Biology Society, Aug 2011.

[8] Islam, S. M. R., Kwak, D., Kabir, M. H., Hossain, M. and Kwak, K. S. The internet of things for health care: A comprehensive survey. IEEE Access, 3,2015 .

[9] van Kranenburg, R. and Bassi, A. IoT challenges. Communications in Mobile Computing, 1(1), Nov 2012.

[10] Darshan, K. and Anandakumar, K. A comprehensive review on usage of internet of things (iot) in healthcare system. In Emerging Research in Electronics, Computer Science and Technology (ICERECT), 2015 International Conference on. IEEE, 2015.

[11] Kulkarni, A. and Sathe, S. Healthcare applications of the internet of things: A review. International Journal of Computer Science and Information Technologies, 5(5), 2014. 